\title{
Setting priorities in the pursuit of universal health coverage
}

Priority setting is fundamental to the fair and efficient pursuit of universal health coverage (UHC). This chapter addresses the key choices in selecting services for UHC and the alternative criteria, tools, and processes to guide these choices. We first describe the choices decision makers have to make on the path to UHC and the recommendations by the WHO Consultative Group on Equity and Universal Health Coverage for how these choices can be made. We next use Thailand as a case study and examine how the Thai government has set priorities in their pursuit of UHC. Against this background, we discuss alternative criteria, tools, and processes for guiding service selection for UHC. When doing this, we consider past experiences in Thailand and other countries, and we examine how recent developments and the insights from the preceding chapters can provide directions for the future.

\section{Hard choices}

UHC is achieved when all people receiving the quality health services they need, without being exposed to financial hardship. ${ }^{1}$ UHC got momentum with the World Health Report $2010^{2}$ and more recently with its inclusion in the Sustainable Development Goals (SDGs). ${ }^{3}$ It is now topping the global health agenda.

However important the goal of UHC is, it cannot be achieved overnight. Judicious priority setting is therefore essential to ensure a fair and efficient path to UHC. The now well-known coverage cube is a useful heuristic to illustrate the key choices for priority setting on that path. The cube depicts 1) the population covered; 2 ) the services made available; and 3) the total costs met from pooled funds.

Figure 1. The coverage cube in World Health $2010^{2}$

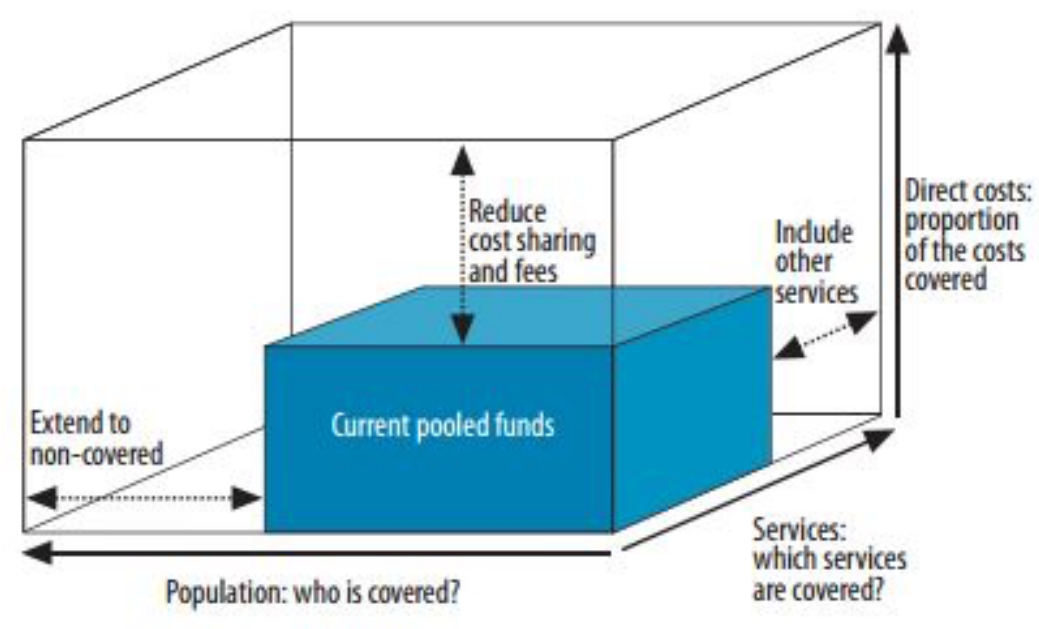

In each dimension, decision makers have to set priorities when moving forward. When expanding services, decision makers have to decide which services to expand first. When including more people, decision makers must decide whom to include first. And when reducing out-of-pocket payments, decision makers must decide how to shift from such payment toward prepayment. On top of this, 
decision makers have to set priorities across dimensions, i.e., they have to trade-off progress in one dimension against progress in others.

Especially the first dimension - selection of services - is at the heart of how "priority setting" is commonly understood. Careful choices in this dimension are also seen as fundamental to the pursuit of UHC. ${ }^{4-6}$ This was stressed by the WHO Consultative Group on Equity and Universal Health Coverage (WHO Consultative Group). ${ }^{7}$ The Group was established in 2012 in response to requests by more than 70 countries for policy support and technical advice for UHC reform from the WHO. The Group recommended a three-part strategy for countries seeking fair progressive realization of UHC:

1) Categorize services into priority classes, using relevant criteria including those related to costeffectiveness, priority to the worse off, and financial risk protection; 2) First expand coverage for high-priority services to everyone, which includes eliminating out-of-pocket payments while increasing mandatory, progressive prepayment with pooling of funds; and 3) While doing this, ensure that disadvantaged groups are not left behind, which often will include low-income groups and rural populations. These three steps need to be regularly repeated, but for each cycle it is clear that priority setting among services is a basic step.

The pivotal role of service selection can be highlighted by looking at current gaps in coverage. Figure 2 shows coverage rates for selected child health interventions in low- and middle-income countries. We see that coverage is far from universal even for these key services. If a decision maker is unable to improve coverage for all these services at once - and these are just some of numerous services competing for resources - he or she has to choose for which one to improve coverage first.

Figure 2. Coverage rates for selected child health interventions in low- and middle-income countries ${ }^{8}$

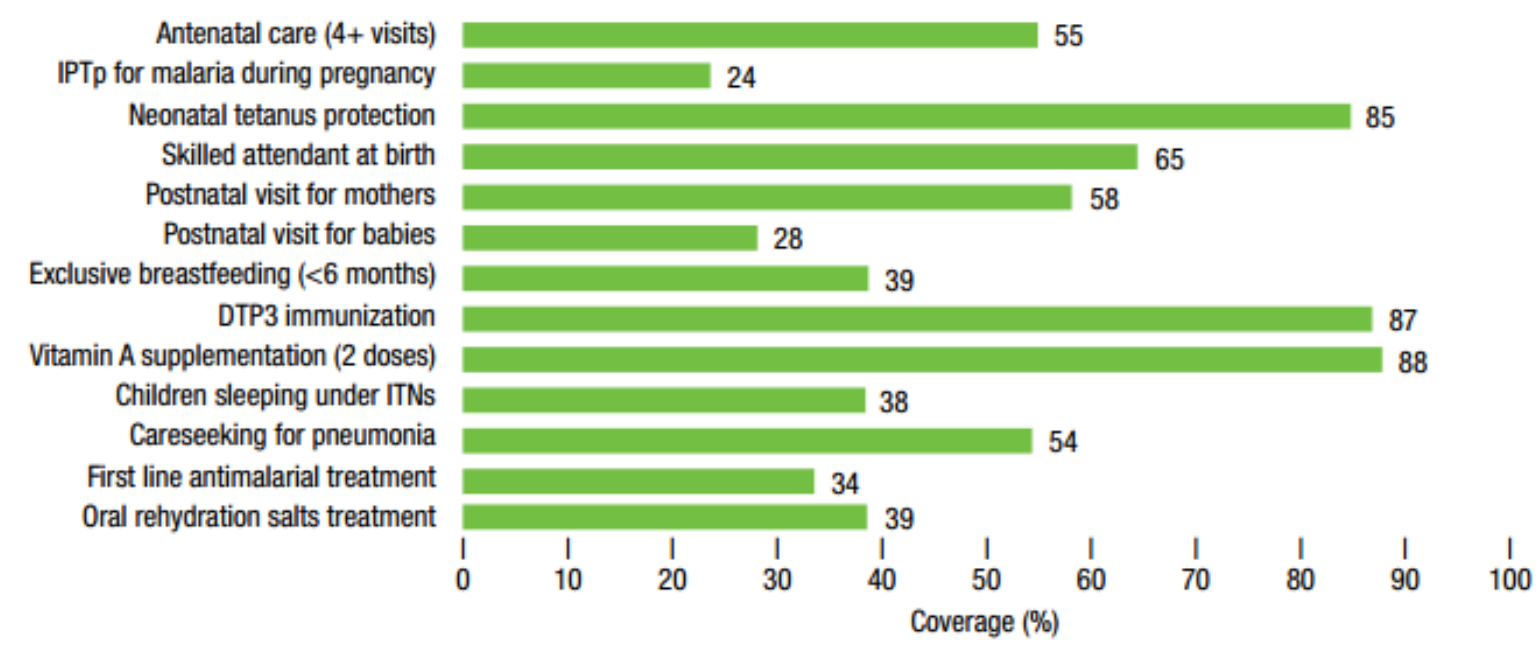

The attention paid to priority setting for UHC in general and service selection for UHC in particular have been limited - some say even neglected. ${ }^{6}$ However, attention has gradually increased. The WHO Consultative Group was established in 2012 to provide guidance on equity and priority setting on the path to UHC, ${ }^{7}$ the International Decision Support Initiative was launched in 2013 with a mandate of supporting policy makers in priority setting for $\mathrm{UHC},{ }^{9}$ and the overarching theme of the 2016 Prince Mahidol Award Conference was "Priority setting for universal health coverage." ${ }^{10}$ 


\section{The case of Thailand}

Many low- and middle-income countries have intensified their pursuit of UHC over the last years, and many have made considerable progress. ${ }^{111}{ }^{11}$ One widely considered frontrunner is Thailand, which has also gradually developed an explicit and systematic approach to priority setting. ${ }^{13-18}$ The case of Thailand thus clearly illustrates some of the key priority-setting choices that countries have to make in their pursuit of UHC, and it also illustrates the kind of criteria, tools, and processes that can guide these choices. At the same time, Thailand is not the best example of a country that chooses to first concentrate on a limited package of services, since the government promised a comprehensive package from early on. However, while the Thai health system is generally considered highly efficient and the gap between de jure and de facto coverage may be smaller than many other places, ${ }^{13}$ there have been some concerns that actual coverage does not entirely match the promises made. ${ }^{19}$

In 2001, the Thai government launched the Universal Coverage Scheme (UCS). ${ }^{14,15}$ This scheme supplemented the Social Security Scheme and the Civil Servant Medical Benefit Scheme and within one year it covered 47 million people, including 18 million people previously uninsured. ${ }^{14}$ The initial benefit package is considered quite comprehensive and included inpatient care (including major surgeries), outpatient care, dental care, and medicines. ${ }^{14-17}$ This package was based on historical precedents and what the other health insurance schemes were covering, ${ }^{14}$ and it was described in terms of both positive and negative lists, where the latter state what interventions are explicitly excluded. ${ }^{16,17}$

The Board of the National Health Security Office was tasked with specifying the benefit package and deciding what new interventions to include. ${ }^{16}$

In 2007, the Subcommittee for the Development of the Benefits Package and Service Delivery was established to offer recommendations to the Board about these decisions. ${ }^{16,17}$ As for the initial benefit package, this committee had to begin with no systematic criteria for selection or rejection of interventions. ${ }^{16}$ One of the Committee's major - and still controversial - recommendations pertained to renal replacement therapy. ${ }^{14,20}$ In the early 2000s, this kind of therapy was not part of the package due to high costs, but nephrologists and kidney patient groups had started to campaign for its inclusion. ${ }^{20}$ In response, the National Health Security Office commissioned research into the options for dialysis. ${ }^{20}$ Compared with palliative care, the incremental cost-effectiveness ratios for initial treatment with peritoneal dialysis and hemodialysis were estimated to 52,000 and 63,000 US\$ (PPP), respectively. ${ }^{21}$ This was generally not considered cost-effective, while it was emphasized that peritoneal dialysis was more cost-effective than hemodialysis. ${ }^{20,}{ }^{22}$ Another study also demonstrated that the budget impact of including renal replacement therapy in the benefit package would be dramatic, accounting for $15 \%$ of the National Health Security Office's budget in the fifteenth year after implementation. ${ }^{23}$ Despite this, in 2007 , the Cabinet finally decided to cover renal replacement theory under the Universal Coverage Scheme. ${ }^{20}$ The reasons for this decision were the financial burdens currently caused by payment for dialysis and the disparities across the three different coverage schemes. ${ }^{19,22}$

However, a more systematic approach was set up in 2009 to help decide what new interventions were to be added to the package, and this approach included five key steps. ${ }^{14,16-18,24}$ First, seven different stakeholder groups, including representatives of patients and the general public, can nominate topics. Second, a topic selection group prioritizes among the proposed topics on the basis of six criteria: 1 ) size of population affected by disease; 2 ) severity of disease in terms of quality of life; 3 ) effectiveness of health intervention; 4) variations in clinical practice across three public health 
insurance schemes; 5) economic impact on household expenditure; and 6) equity, ethical, and social implications. ${ }^{18}$ Third, the International Health Policy Program or the Health Intervention and Technology Assessment Program (HITAP) assess the prioritized topics in terms of their costeffectiveness and budget impact. Fourth, the results of this assessment is presented to the Subcommittee for Development of the Benefit Package and Service Delivery, which then provides policy recommendations for the Board of the National Health Security Office. And fifth, the Board makes the final decision.

Since the adoption of this more systematic approach in 2009, the Subcommittee has recommended the inclusion of many new interventions, while rejecting the inclusion some others. ${ }^{14,16-18,25}$ Among the interventions accepted are screening for Downs syndrome, treatment of multidrug-resistant tuberculosis, treatment of severe thalassemia by implantation, and a smoking cessation program. ${ }^{17,18}$ Eleven new population-based screening programs have also been accepted for inclusion from 2016, including diabetes screening using fasting plasma glucose for those aged 30 years and over every 5 years and cervical cancer screening using pap smear or visual inspection by acetic acid for women $30-60$ years old every 5 years. ${ }^{25}$

Among the interventions that have been rejected are pegylated interferon alpha-2a with ribavirin for treating hepatitis $\mathrm{C}$, absorbent products for urinary and fecal incontinence among disabled and elderly people, and anti-immunoglobulin $\mathrm{E}$ for severe asthma. ${ }^{18}$ The latter was rejected because it was not considered cost-effective and the budget impact was judged too high. ${ }^{18}$ The first two interventions were considered cost-effective, but rejected because of the budget impact was seen as too high. ${ }^{18}$ Among the population-based screening programs that have been rejected are chest $\mathrm{x}$-ray for asymptomatic tuberculosis (during routine check-up) due to evidence of possible harm; urinary analysis and renal functions testes for nephritis, nephrosis, and renal calculi (during routine checkup) due to lack of evidence on effectiveness; and mammography for screening for breast cancer in women aged $40-59$ years because of it was not found cost-effective.$^{25}$

\section{Criteria and tools}

When selecting services in the pursuit of UHC, a fundamental issue is what criteria to use and how to balance them. ${ }^{7,11}$ Explicit, concrete criteria can help decision makers in making hard choices, help ensure that decisions are consistent across cases, and promote transparency and accountability. ${ }^{7,26}$ Thailand, for example, uses six criteria to guide the initial selection of topics and other criteria to assess interventions in more depth. Another example is found in Mexico; also a country well known for its strides towards UHC and its systematic approach to priority setting. ${ }^{27-29}$ Its Fund for Protection Against Catastrophic Health Expenditures includes new interventions in the package on the basis of cost-effectiveness, affordability, financial protection, opinion of the scientific community, demand and supply, and social acceptance. ${ }^{28}$ Many countries are not so explicit about what criteria they use, and among those that are, there is considerable variation in which criteria they emphasize. ${ }^{11,13} \mathrm{~A}$ World Bank review of UHC programs found that the most common criteria were affordability, costeffectiveness, financial protection, and opinion of the scientific community, in that order. ${ }^{11}$

Some criteria are associated with particular decision-support tools. A cost-effectiveness criterion, for example, is often linked to formal cost-effectiveness analysis, although the criterion can be useful also when applied more loosely. Similarly, criteria related to affordability and societal health needs can be linked to rigorous budget-impact and disease-burden analyses, respectively. ${ }^{30,31}$ However, the approaches taken are often not so rigorous, ${ }^{32}$ and criteria related to severity of disease and financial 
protection have also tended to be applied on a more discretionary basis. Some countries also use specific tools to balance different criteria, including multi-criteria decision analysis. ${ }^{33}$ The Thai government, for example, employs cost-effectiveness analysis, budget-impact analysis, diseaseburden analysis, and then a form of multi-criteria decision analysis with deliberation to reach an overall judgment.

The WHO Consultative Group emphasized three criteria for service selection, while allowing for other criteria to also be relevant. The three criteria were related to cost-effectiveness, priority to the worse off, and financial risk protection, respectively. The Group further described how each of these criteria could be linked to tools and methods for quantitative assessment and how the criteria could be traded off against each other through systematic approaches. From this perspective, it is interesting to examine how countries currently relate to the three criteria emphasized by the WHO Consultative Group, what challenges the countries' face, and how recent developments may facilitate progress.

\section{Cost-effectiveness}

Many countries use cost-effectiveness as a criterion to guide service selection for UHC and systematically integrate data on cost-effectiveness in their decision-making processes. ${ }^{11}$ The Universal Coverage Scheme in Thailand and the Popular Health Insurance (Seguro Popular) in Mexico are just two examples. ${ }^{16,28}$ However, many other countries still do not seem to emphasize costeffectiveness in service selection or systematically use cost-effectiveness data. ${ }^{11,34}$ Those that do, face several challenges that are often particularly pronounced in low- and middle-income countries, including limitations in data and in capacity to conduct and interpret cost-effectiveness analyses. ${ }^{32,34-}$ ${ }^{36}$ In addition, cost-effectiveness criteria and cost-effectiveness analyses tend to focus on health outcome measures and often quality-adjusted or disability-adjusted life years more specifically. ${ }^{34-37}$ Many stakeholders also consider other, non-health outcomes as relevant for service selection, including educational benefits, productivity gains, and financial risk protection. ${ }^{11,38-40}$

Over the last years, support has continued to build up for the case that countries should systematically consider cost-effectiveness when selecting services in the pursuit of UHC. This support has come not only from economists, but also from a broad range of other actors and disciplinary perspectives. For example, the WHO Consultative Group argued that cost-effectiveness considerations are crucial for making fair progress towards UHC. It has also recently been argued that such considerations are important from a right-to-health perspective, with the caveat that costeffectiveness criterion must not be used to justify UHC below the minimum level demanded by the right to health. ${ }^{41}$

The situation with regard to data and tools is also improving. While the share of cost-effectiveness analyses conducted in low- and middle-income countries is still low, ${ }^{35}$ more attention has been directed towards the generation and use of cost-effectiveness information in these countries in recent years, and the number of analyses have been steadily growing. ${ }^{36}$ The generation and use of cost-effectiveness data have also been promoted by the ongoing WHO-CHOICE project; the basis of which has been thoroughly examined in this volume (chapter [insert chapter number of chapter by Lauer et al]). Similarly, the use and generation of cost-effectiveness have been advanced by the International Decision Support Initiative, ${ }^{9}$ including as part of health technology assessments, and the third edition of the Disease Control Priorities project, which provides evidence on intervention efficacy and program effectiveness for the leading causes of global disease burden..$^{42}$ At the same time, new methods have been developed for defining cost-effectiveness thresholds in low- and middle-income countries. ${ }^{43}$ These methods go beyond the generic WHO-thresholds as multiples of 
GDP per capita and thresholds based on willingness-to-pay studies and seek to estimate thresholds that reflect the opportunity cost of implementing the intervention being considered. While there is still disagreement about how such thresholds should be calculated, they may become useful for assisting priority setting and helping define benefit packages. ${ }^{44,45}$

\section{Worse off}

A special concern for the worse off or disadvantaged individuals or groups is often integral to the pursuit of UHC, although specific focus and terminology vary widely. ${ }^{7,46}$ The worse off can be defined in terms of health, socio-economic status, and several other metrics. ${ }^{7,39,46,47}$ With respect to health, the concern for the worse off are often framed in terms of individual need, severity, or urgency. ${ }^{7,39,40}$

Despite the central role of the concern for the worse off in motivating UHC, this concern is rarely operationalized as a distinct criterion for service selection in low- and middle-income countries. ${ }^{11,32}$ Thailand is an exception, where at least two criteria for prioritizing among proposed topics can be seen as linked to a concern for the worse off. One criterion is severity, which assigns extra priority to interventions targeting the severely ill because of their greater need for health care.$^{18}$ Another is a criterion linked to equity and ethical implications, which, among other things, assigns extra priority to interventions targeting diseases that are more frequent among the poor. ${ }^{18}$

One reason why the concern for the worse off is rarely operationalized as a separate criterion may be a belief that a cost-effectiveness criterion will point towards the same interventions, although there are numerous examples of where this is not the case [chapter number of chapter by Norheim, Johansson, and Ottersen]. A more important reason, however, is likely to be that the concern for the worse off is seen as particularly difficult to operationalize in a way that both captures the relevant dimensions and allow for measurement based on available data. In Thailand, for example, the severity criterion has been omitted in some evaluations because the information for the interventions in question was seen as limited and incomparable. ${ }^{18}$

Despite this, the case for a service-selection criterion directly linked to the worse off has been made gradually stronger over the years. In 2013, the Lancet Commission on Investing in Health argued for prioritizing services targeting conditions that disproportionally affect the poor as part of one of their two "progressive pathways" to UHC. ${ }^{48}$ The year after, the WHO Consultative Group argued for priority to services benefiting the worse off more generally, while focusing individuals and groups with the poorest health outcomes. Priority to the worse off also has a firm grounding in the theory of fair distribution, as is discussed in chapter [chapter number of chapter by Voorhoeve] in this volume, and surveys suggest that the judgment that the worse off should receive some priority is broadly shared across societies. ${ }^{49-51}$

To operationalize a criterion directly linked to priority to the worse off may also be more doable than is commonly thought, including a criterion linked to the worse off in terms of health. Various QALYloss criteria have recently been developed and considered in several high-income countries, including the UK, the Netherlands, and Norway. ${ }^{52}$ These criteria assign priority to interventions that benefit individuals or groups that without intervention would lose a large number of QALYs due to their condition. It has been demonstrated how QALY-loss can be approximated using data underlying economic evaluations of interventions or burden-of-disease studies. ${ }^{52,53}$ There has also been developments with regard to systematic approaches for balancing a worse-off criterion against other criteria. This include approaches that assign weights to cost-effectiveness ratios based on how badly off the beneficiary is, as is further discussed in chapter [chapter number of chapter by Norheim, Johansson, and Ottersen] in this volume. These two concerns can also be sought balanced through 
the use of extended cost-effectiveness analysis or benefit-cost analysis; methods that also are discussed in this volume (chapters [chapter numbers for chapter by Verguet and by Robinson]) Finally, many types of multi-criteria decision analyses have been developed and tested over the last years, and these offer another way to balance cost-effectiveness and priority to the worse off. ${ }^{33}$

\section{Financial risk protection}

Service selection in the context of UHC is typically a question of what services should be financed mainly or entirely by mandatory, prepaid, pooled funds, which may be called social financing for short. Social financing of one service may provide more financial risk protection than social financing of another, and some countries uses a service-selection criterion that assigns extra priority to services whose coverage offers substantial financial risk protection. ${ }^{11}$ As described above, one of these countries is Thailand, which use a criterion linked to the economic impact of health expenditure on household expenditure. ${ }^{18}$ Another is Mexico, whose designated Fund for Protection Against Catastrophic Health Expenditures uses financial protection as a central criterion for deciding what interventions to include. ${ }^{28} \mathrm{At}$ the same time, many other countries using explicit criteria to select services do not include such a criterion linked to financial risk protection.

One reason for the hesitance may be some form of "health exceptionalism" or a view that health care is special. ${ }^{47,54}$ For example, one may believe that priority setting among health services should depend on medical factors only and that the financial burden linked to health service payments is not such a factor. Another reason may be the challenges involved with operationalizing one or more criteria linked to financial risk protection. ${ }^{55}$ This is underscored by the heated debate about indicators for financial risk protection for the SDGs. ${ }^{56}$ Moreover, approaches for integrating a criterion linked to financial risk protection with other criteria have been explored only to a limited extent.

Yet, the case for a service-selection criterion linked to financial risk protection is particularly strong in the context of UHC, as it can be seen as a dual goal: to ensure access to health services and to ensure that people do not face large economic hardships when paying for services. ${ }^{57}$ It is the latter and not the former that is commonly seen as the distinguishing feature of the UHC agenda compared to earlier agendas, including "health for all." Forceful arguments have also recently been put forward in favor of going beyond medical factors when selecting services, ${ }^{47}$ including in chapter [chapter number of chapter by Persad] in this volume.

Over the last years, there has also been progress on the measurement of financial risk protection linked to payment for services. Indicators of the incidence of catastrophic and impoverishing health expenditure have been developed and used, including in the first global UHC monitoring report in 2015. ${ }^{1}$ Some UHC indices - integrating tracer indicators for both service coverage and financial protection - have also been developed. ${ }^{12,58}$ This work with indicators and indices can help operationalize a service-selection criterion linked to financial risk protection. To integrate such a criterion with other criteria, the recent development and use of ECEA and BCA can be helpful, as discussed in chapters [insert chapter numbers of chapters by Verguet and Robinson]. As for a criterion linked to a concern for the worse off, recent development and use of multi-criteria decision analysis can be useful for incorporating a criterion linked to financial protection. ${ }^{59}$ Thailand, for example, uses such an analysis to integrate both these kinds of criteria. 


\section{Process}

In countries where explicit criteria and tools are used, they are part of a larger decision-making process. $^{7,11,32,60-63}$ This process itself can be more or less explicit and systematic, and some low- and middle-income countries have set up quite rigorous processes for selecting services on the path to UHC, including Thailand and Mexico. ${ }^{28,32,62}$

As for criteria and tools, countries face some common challenges when seeking to introduce and run systematic and fair priority-setting processes. One challenge is the political economy of priority setting. ${ }^{63-65}$ Priority setting is controversial and comes with negative connotations. Making the process explicit can thus reflect poorly on those seen as responsible, including politicians. Priority setting is also an area with powerful vested interests. Pharmaceutical companies, patient groups, and professional groups may all prefer that at least parts of the priority-setting process are vague and unsystematic. A rigorous process also require considerable institutional capacity. ${ }^{29,66}$ Technical knowhow and information-technology infrastructure are needed for the generation and assessment of large amounts of scientific evidence, and at multiple points in the process, mechanisms must be in place to carefully integrate evidence and value. It is also widely agreed that participation from a broad range of stakeholders is essential to a good and fair priority-setting process, and ensuring that all relevant stakeholders are included in a meaningful way may require a quite robust institutional framework. As for criteria and tools, recent developments have prepared the ground for progress also on process. The issue of priority-setting processes and institutions in low- and middle-income countries have received quite some attention over the last few years, and especially around and after the 2016 Prince Mahidol Award Conference..$^{10}$ This has partly taken place in the context of research and debate on health technology assessment in resource-poor settings. ${ }^{67}$ Several other trends also bode well for strengthening priority-setting processes in the future. The political economy of priority setting in global health has recently attracted more attention, ${ }^{63-65}$ and the issue of accountability is experiencing renewed interest. At the same time, the issue of public participation in priority setting has steadily come more to the fore. It was highlighted by the WHO Consultative Group and is examined in more depth in chapter [insert chapter number of chapter by McCoy] in this volume. For the future of priority setting and UHC, it is important that these issues continue to receive attention. In addition, two other long-standing issues will also need further scrutiny. One is the upstream process of agreeing on criteria to use in the selection of services. In Thailand, for example, the guidelines for the procedure of selecting services were themselves developed through an elaborate process, which included consultations with a broad range of stakeholders. ${ }^{24} \mathrm{~A}$ final, fundamental issue is the relationship between substantive criteria and the process for service selection. ${ }^{26,68}$ Once the criteria are determined, how much and what should be left up to the process? A clear understanding of the pros and cons with different answers to this question will help future priority setting.

\section{Conclusions}

This chapter has addressed the key choices in selecting services for UHC and the alternative criteria, tools, and processes to guide these choices. Over the last years, many countries have made progress towards UHC and also towards more systematic priority setting in support of that larger endeavor. Yet, many countries still lack explicit criteria, rigorous tools, and systematic processes, and a set of common challenges applies to most low- and middle-income countries. At the same time, recent 
developments - including insights from the preceding chapters of this volume - can help countries meet these challenges and improve their approach to priority setting. This will be critical for effectively achieving UHC and health lives for all, and for doing so fairly.

\section{References}

1. World Health Organization, World Bank. Tracking universal health coverage: First global monitoring report. Geneva: WHO; 2015.

2. World Health Organization. The World Health Report 2010. Health systems financing: The path to universal coverage. Geneva: 2010.

3. United Nations General Assembly resolution 70/1. Transforming our world: The 2030 Agenda for Sustainable Development 2015.

4. Chalkidou K, et al. Priority-setting for achieving universal health coverage. Bull World Health Organ. 2016;94:462-7.

5. Reich MR. Introduction to the pmac 2016 special issue: "Priority setting for universal health coverage". Health Systems \& Reform. 2016;2:1-4.

6. Vega J, Frenz P. Latin America: Priorities for universal health coverage. Lancet. 2015;385:e312.

7. World Health Organization. Making fair choices on the path to universal health coverage. Final report of the WHO Consultative Group on Equity and Universal Health Coverage. Geneva: 2014.

8. World Health Organization. World health statistics 2016: Monitoring health for the SDGs, sustainable development goals. Geneva: WHO; 2016.

9. International Decision Support Initiative. Better decisions. Better health. Available from: http://www.idsihealth.org/ [14.08.16].

10. Prince Mahidol Award Conference. Priority setting. Available from: http://www.pmaconference.mahidol.ac.th/index.php?option=com_content\&view=article\&id $=743 \&$ Itemid $=213$ [14.08.16].

11. Cotlear D, et al. Going universal: How 24 developing countries are implementing universal health coverage reforms from the bottom up. Washington, DC: World Bank; 2015.

12. Wagstaff $A$, et al. Measuring progress towards universal health coverage: With an application to 24 developing countries. Ox Rev of Eco Policy. 2016;32:147-89.

13. Bredenkamp C, et al. Emerging challenges in implementing universal health coverage in Asia. Soc Sci Med. 2015;145:243-8.

14. Thailand's universal coverage scheme: Achievements and challenges. An independent assessment of the first 10 years (2001-2010). Nonthaburi: Health Insurance System Research Office; 2012.

15. Hanvoravongchai P. Health financing reform in thailand: Toward universal coverage under fiscal constraints. Universal Health Coverage Studies Series No 20. Washington, DC: World Bank; 2013.

16. Culyer AJ, et al. A star in the east: A short history of hitap. Nonthaburi: Health Intervention and Technology Assessment Program; 2016.

17. Jongudomsuk P, et al. Evidencebased health financing reform in thailand. In: B C, D C, S G, eds. The economics of public health care reform in advanced and emerging economies. Washington, DC: International Monetary Fund; 2012. p. 307-26.

18. Youngkong $\mathrm{S}$, et al. Multicriteria decision analysis for including health interventions in the universal health coverage benefit package in thailand. Value in Health. 2012;15:961-70.

19. Tangcharoensathien $\mathrm{V}$, et al. Promoting universal financial protection: How the thai universal coverage scheme was designed to ensure equity. Health Res Policy Syst. 2013;11:25. 
20. Tantivess $S$, et al. Universal coverage of renal dialysis in thailand: Promise, progress, and prospects. BMJ. 2013;346:f462.

21. Teerawattananon $\mathrm{Y}$, et al. Economic evaluation of palliative management versus peritoneal dialysis and hemodialysis for end-stage renal disease: Evidence for coverage decisions in thailand. Value in Health. 2007;10:61-72.

22. Patcharanarumol $W$, et al. Why and how did thailand achieve good health at low cost? In: Balabanova D, McKee M, Mills A, eds. Good health at low cost' 25 years on: What makes a successful health system? London: London School of Hygiene and Tropical Medicine; 2011. p. 193-223.

23. Kasemsup $\mathrm{V}$, et al. An estimate of demand for rrt under universal healthcare coverage in thailand. Journal of the Nephrology Society of Thailand. 2006;12:125-35.

24. Tantivess $S$, et al. Efficiency or equity: Value judgments in coverage decisions in thailand. $J$ Health Organ Manag. 2012;26:331-42.

25. Teerawattananon $Y$, et al. Development of a health screening package under the universal health coverage: The role of health technology assessment. Health Econ. 2016;25 Suppl 1:162-78.

26. Daniels N, Sabin JE. Setting limits fairly: Learning to share resources for health. 2nd ed. New York: Oxford University Press; 2008.

27. Knaul FM, et al. The quest for universal health coverage: Achieving social protection for all in Mexico. Lancet. 2012;380:1259-79.

28. Bonilla-Chacín ME, Aguilera N. The mexican social protection system in health. Universal Health Coverage Studies Series No 1. Washington, DC: World Bank; 2013.

29. Gonzalez-Pier E, et al. Priority setting for health interventions in mexico's system of social protection in health. Lancet. 2006;368:1608-18.

30. Mauskopf JA, et al. Principles of good practice for budget impact analysis: Report of the ispor task force on good research practices--budget impact analysis. Value Health. 2007;10:336-47.

31. Murray CJ, et al. Global, regional, and national disability-adjusted life years (DALYs) for 306 diseases and injuries and healthy life expectancy (HALE) for 188 countries, 1990-2013: Quantifying the epidemiological transition. Lancet. 2015;386:2145-91.

32. Giedion U, et al., eds. Health benefit plans in Latin America: A regional comparison. Washington, DC: Inter-American Development Bank; 2014.

33. Cromwell I, et al. 'Real-world' health care priority setting using explicit decision criteria: A systematic review of the literature. BMC Health Serv Res. 2015;15:164.

34. Griffiths UK, et al. Comparison of economic evaluation methods across low-income, middleincome and high-income countries: What are the differences and why? Health Econ. 2016;25 Suppl 1:29-41.

35. Pitt $\mathrm{C}$, et al. Economic evaluation in global perspective: $\mathrm{A}$ bibliometric analysis of the recent literature. Health Econ. 2016;25 Suppl 1:9-28.

36. Pitt $\mathrm{C}$, et al. Foreword: Health economic evaluations in low- and middle-income countries: Methodological issues and challenges for priority setting. Health Econ. 2016;25 Suppl 1:1-5.

37. Greco G, et al. Outcomes in economic evaluations of public health interventions in low- and middle-income countries: Health, capabilities and subjective wellbeing. Health Econ. 2016;25 Suppl 1:83-94.

38. van Mastrigt GA, et al. A qualitative study on the views of experts regarding the incorporation of non-health outcomes into the economic evaluations of public health interventions. BMC public health. 2015;15:954.

39. Guindo LA, et al. From efficacy to equity: Literature review of decision criteria for resource allocation and healthcare decisionmaking. Cost Eff Resour Alloc. 2012;10:9.

40. Norheim OF, et al. Guidance on priority setting in health care (GPS health): The inclusion of equity criteria not captured by cost-effectiveness analysis. Cost Eff Resour Alloc. 2014;12:18.

41. World Health Organization. Anchoring universal coverage in the right to health: What difference would it make? Geneva: WHO; 2015. 
42. University of Washington. Dcp3: Disease control priorities. Available from: http://dcp-3.org/ [14.08.16].

43. Ochalek J, et al. Cost per daly averted thresholds for low- and middle-income countries: Evidence from cross country data. CHE Research Paper 122. 2015.

44. Culyer AJ. Cost-effectiveness thresholds in health care: A bookshelf guide to their meaning and use. Health Econ Policy Law. 20161-18.

45. Claxton $\mathrm{K}$, et al. Methods for the estimation of the national institute for health and care excellence cost-effectiveness threshold. Health Tech Assess. 2015;19.

46. Rockefeller Foundation, Save the Children, UNICEF, WHO. Universal health coverage: A commitment to close the gap. London: Save the Children; 2013.

47. Sharp D, Millum J. Prioritarianism for global health investments: Identifying the worst off. Journal of Applied Philosophy. 2015;Online first.

48. Jamison DT, et al. Global health 2035: A world converging within a generation. Lancet. 2013;382:1898-955.

49. Dolan P, et al. QALY maximisation and people's preferences: A methodological review of the literature. Health Economics. 2005;14:197-208.

50. Youngkong $S$, et al. Setting priorities for health interventions in developing countries: A review of empirical studies. Tropical Medicine and International Health. 2009;14:930-9.

51. Kapiriri L, Norheim OF. Criteria for priority-setting in health care in Uganda: Exploration of stakeholders' values. Bulletin of the World Health Organization. 2004;82:172-9.

52. Ottersen T, et al. A new proposal for priority setting in Norway: Open and fair. Health Policy. 2016;120:246-51.

53. Claxton K, et al. Causes for concern: Is nice failing to uphold its responsibilities to all NHS patients? Health Econ. 2015;24:1-7.

54. Segall S. Is health care (still) special? Journal of Political Philosophy. 2007;15:342-61.

55. Ruger JP. An alternative framework for analyzing financial protection in health. PLoS Med. 2012;9:e1001294.

56. Mariott A, Kamal-Yanni M. Last minute change to the uhc indicator for the SDGs is raising alarm bells! Global Health Check. 2016 07.03.16.

57. Jha A, et al. Delivering on the promise of universal health coverage. BMJ. 2016;353:i2216.

58. Prinja $S$, et al. A composite indicator to measure universal health care coverage in India: Way forward for post-2015 health system performance monitoring framework. Health Policy Plan. 2016.

59. Marsh K, et al. Multiple criteria decision analysis for health care decision making--emerging good practices: Report 2 of the ispor mcda emerging good practices task force. Value Health. 2016;19:125-37.

60. Baltussen $\mathrm{R}$, et al. Priority setting for universal health coverage: We need evidence-informed deliberative processes, not just more evidence on cost-effectiveness. International Journal of Health Policy and Management. 2016;5:1-4.

61. Glassman A, et al. Defining a health benefits package: What are the necessary processes? Health Systems \& Reform. 2016;2:39-50.

62. Glassman A, Chalkidou K. Priority-setting in health: Building institutions for smarter public spending. Washington, DC: Center for Global Development; 2012.

63. Kieslich K, et al. Accounting for technical, ethical, and political factors in priority setting. Health Systems \& Reform. 2016;2:51-60.

64. Hauck K, Smith P. The politics of priority setting in health: A political economy perspective. Working Paper 414. Center for Global Development; 2015.

65. Gupta V, et al. Politics and universal health coverage - the post-2015 global health agenda. New England Journal of Medicine. 2015;373:1189-92.

66. Chootipongchaivat $\mathrm{S}$, et al. Factors conducive to the development of health technology assessment in Asia: Impacts and policy options. Policy brief. Geneva: World Health Organization; 2015. 
67. Teerawattananon Y, Luz A. Health technology assessment as a priority-setting tool for universal health coverage: The call for global action at the prince mahidol award conference 2016. Pharmacoeconomics. 2016;34:1-3.

68. Rumbold BE, Wilson J. Reasonable disagreement and the generally unacceptable: A philosophical analysis of making fair choices. Health Econ Policy Law. 2016;11:91-6. 